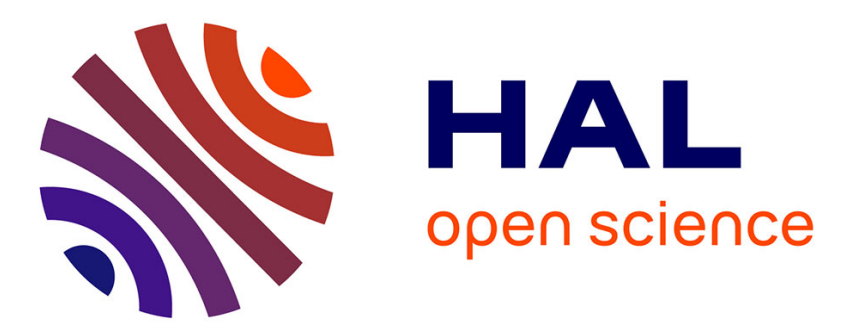

\title{
Improving MIMO detection performance in presence of phase noise using norm difference criterion
}

\author{
Tanumay Datta, Sheng Yang
}

\section{To cite this version:}

Tanumay Datta, Sheng Yang. Improving MIMO detection performance in presence of phase noise using norm difference criterion. 53rd Annual Allerton Conference on Communication, Control, and

Computing, Sep 2015, Monticello, IL, United States. 10.1109/allerton.2015.7447017 . hal-01261219

\section{HAL Id: hal-01261219 \\ https://hal-centralesupelec.archives-ouvertes.fr/hal-01261219}

Submitted on 24 Jan 2016

HAL is a multi-disciplinary open access archive for the deposit and dissemination of scientific research documents, whether they are published or not. The documents may come from teaching and research institutions in France or abroad, or from public or private research centers.
L'archive ouverte pluridisciplinaire HAL, est destinée au dépôt et à la diffusion de documents scientifiques de niveau recherche, publiés ou non, émanant des établissements d'enseignement et de recherche français ou étrangers, des laboratoires publics ou privés. 


\title{
Improving MIMO detection performance in presence of phase noise using norm difference criterion
}

\author{
Tanumay Datta and Sheng Yang \\ Centrale Supélec, Gif-sur-Yvette, France
}

\begin{abstract}
Practical MIMO communication systems suffer performance loss from oscillator phase noise. In particular, traditional maximum likelihood (ML) detection algorithm results in an error floor in symbol error probability, and thus becomes unable to harvest the spatial diversity to be obtained in MIMO systems without phase noise. In this paper, we propose a method to detect the correctness of the traditional ML solution in the presence of strong phase noise. A criteria based on the ML cost differences between the ML solution and the next best solutions is used to determine a set of possible candidate solutions. We also propose a novel algorithm for data detection using phase noise estimation techniques to obtain an modified ML cost for each of the candidate solutions. This approach results in symbol error rate performance improvement by reducing the error floor without incurring much additional complexity due to phase noise estimation. Theoretical arguments as well as simulation studies are presented to support the performance improvement achieved.
\end{abstract}

\section{INTRODUCTION}

¡iGeneric introductory material about MIMO, phase noise, literature to be $\operatorname{added}_{i} i$

The main motivation in this paper is to use the traditional ML detection solution which is obtained easily for small MIMO systems to get a better detection performance in presence of phase noise. Our main contribution here is two-fold. Firstly, we have studied the properties of ML cost function in MIMO systems in presence of phase noise. We formulate a criterion based on norm differences between the best and the second best vectors to decide upon the correctness of the ML solution. Next using this criterion we generate a set of candidate vectors for further processing to improve the solution. Theoretical arguments have been presented for the norm difference criterion. Secondly, we formulate a modified ML cost function which accounts for the transmit and receive phase noise values as well. Next for each of the candidate vectors this modified ML cost is computed by estimating phase noise values iteratively. The vector that minimizes the modified ML cost among the candidate set of vectors is declared as the final solution. This proposed detection algorithm is shown to have performance improvement in terms of SER without incurring much additional complexity due to phase noise estimation. SER simulation results for various antenna, modulation alphabet combinations are presented. For example, in a $4 \times 4$ MIMO system using 4-quadrature amplitude modulation (QAM) alphabet in high SNR regime, 85\% SER improvement is obtained while incurring extra phase noise estimation complexity in only $7 \%$ of cases. Although simulation results are only shown for MIMO wireless channel models, the pro- posed techniques are applicable for other types of channels also which suffer from phase noise phenomenon.

The rest of the paper is organized as follows. In section II, we describe the MIMO channel model with phase noise. The study of properties of ML cost and the generation of candidate set of vectors based on norm difference criterion in presented in section III. In section IV, we present the proposed detection algorithm using phase noise estimation techniques. The simulation results and discussions are presented in section V. Finally the paper is concluded in section VI.

\section{Channel Model}

We assume a MIMO wireless channel with $N_{t}$ transmit and $N_{r}$ receive antennas. The transmitted vector is denoted by $\mathbf{x}=\left[x_{1}, x_{2}, \cdots, x_{N_{t}}\right]^{T}$, where $x_{k} \in \mathcal{C}, 1 \leq k \leq N_{t}$. Let $E_{s}$ denote the average energy of the alphabet $\mathcal{C}$. In this paper, we only consider constellations as symbol alphabet with uniform probability distribution over the points. Let $\mathbf{H}$ denote the channel matrix, where the $(i, k)$ th element of $\mathbf{H}$, denoted as $h_{i, k}$, represents the channel gain between $k$ th transmit antenna and $i$ th receive antenna. We assume Rayleigh-fading wireless channel, hence $h_{i, k} \sim \mathbb{C N}(0,1) \forall i, k$. The received vector in base-band $\mathbf{y}$ is defined as

$$
\mathbf{y}=\Theta_{R} \mathbf{H} \Theta_{T} \mathbf{x}+\mathbf{n},
$$

where $\Theta_{R}$ and $\Theta_{T}$ denotes the phase noise matrices on receive and transmit side respectively, and $\mathbf{n}$ is the additive white Gaussian noise (AWGN) vector whose $i$ th entry $n_{i} \sim$ $\mathbb{C} \mathcal{N}\left(0, N_{0}\right), 1 \leq i \leq N_{r}$. The average received signal-tonoise ratio (SNR) is defined as $\gamma=10 \log _{10} \frac{N_{t} E_{s}}{N_{0}}$ in $\mathrm{dB}$.

The transmit and receive phase noise matrices are defined as $\boldsymbol{\Theta}_{T}=\left[\operatorname{diag}\left(e^{j \theta_{t, 1}}, e^{j \theta_{t, 2}}, \cdots, e^{j \theta_{t, N_{t}}}\right)\right]$ and $\boldsymbol{\Theta}_{R}=$ $\left[\operatorname{diag}\left(e^{j \theta_{r, 1}}, e^{j \theta_{r, 2}}, \cdots, e^{j \theta_{r, N_{r}}}\right)\right]$. We also define the vector form representation of all the transmit and receive noise values as $\bar{\theta}=\left[\theta_{t, 1}, \theta_{t, 2}, \cdots, \theta_{t, N_{t}}, \theta_{r, 1}, \theta_{r, 2}, \cdots, \theta_{r, N_{r}}\right]^{T}$. All the oscillators are assumed to be independent and identical. We also assume that $\theta_{t, k} \sim \mathcal{N}\left(0, \sigma_{t}^{2}\right), 1 \leq k \leq N_{t}$ and $\theta_{r, i} \sim \mathcal{N}\left(0, \sigma_{r}^{2}\right), 1 \leq i \leq N_{r}$. For simplicity we assume that $\sigma_{t}^{2}=\sigma_{r}^{2}=\sigma_{\theta}^{2}$. In this paper, we consider the scenario when perfect channel state information in available in the receiver (CSIR), i.e., $\mathbf{H}$ is known at the receiver. Also, the statistics of AWGN and phase noise vectors (i.e., $N_{0}$ and $\sigma_{\theta}^{2}$ respectively) are known at the receiver. We also denote $\mathbf{H}_{\theta}=\boldsymbol{\Theta}_{R} \mathbf{H} \Theta_{T}$ and $\mathbf{R}=\mathbf{H}^{H} \mathbf{H}$. 


\section{Properties of ML COST IN PRESENCE OF PHASE NOISE}

In this section we study the properties of ML cost function for MIMO detection in presence of phase noise and formulate techniques to approach the problem of data detection in such channels. The ML solution can be written as

$$
\begin{aligned}
\mathbf{x}_{M L} & =\arg \max _{\widetilde{\mathbf{x}} \in \mathcal{C}^{N_{t}}} p(\widetilde{\mathbf{x}} \mid \mathbf{y}) \\
& =\arg \max _{\widetilde{\mathbf{x}} \in \mathcal{C}^{N_{t}}} p(\mathbf{y} \mid \widetilde{\mathbf{x}}) \\
& =\arg \max _{\widetilde{\mathbf{x}} \in \mathcal{C}^{N_{t}}} \int_{\widetilde{\theta}} p(\mathbf{y} \mid \widetilde{\mathbf{x}}, \widetilde{\theta}) p(\widetilde{\theta}) d \widetilde{\theta}
\end{aligned}
$$

It can be noted that the evaluation of the ML solution in presence of phase noise is not straightforward. But as an suboptimum technique the receiver can perform traditional $\mathrm{ML}$ detection using $\mathbf{H}$ instead of $\mathbf{H}_{\theta}$ ignoring phase noise, as $\mathbf{H}$ is perfectly known at the receiver but not $\mathbf{H}_{\theta}$. We define $f(\widetilde{\mathbf{x}})=\|\mathbf{y}-\mathbf{H} \widetilde{\mathbf{x}}\|^{2}$ as the ML cost of a vector $\widetilde{\mathbf{x}}$ obtained ignoring phase noise and will referred to as 'impure' ML cost function. Let us define

$$
\mathbf{x}_{M L}^{(1)}=\arg \min _{\widetilde{\mathbf{x}} \in \mathcal{C}^{N_{t}}}\|\mathbf{y}-\mathbf{H} \widetilde{\mathbf{x}}\|^{2},
$$

which is the impure ML solution. It can be observed that, $\mathbf{x}_{M L}^{(1)}=\mathbf{x}_{M L}$ when $\sigma_{\theta}=0$. Also, if $\sigma_{\theta}=N_{0}=0$, then $\mathbf{x}_{M L}^{(1)}=\mathbf{x}_{M L}=\mathbf{x}$, i.e. the true ML solution and 'impure' ML solution coincides with the actually transmitted vector. But in general, they may or may not be same. Our main aim in this section is to find criterion for $\mathbf{x}_{M L}^{(1)}=\mathbf{x}$, and if the criterion is not met, find a set of candidate vectors which contains $\mathbf{x}$.

In absence of phase noise, i.e. $\sigma_{\theta}=0$, the ML cost of the actual transmitted vector is nothing but the norm of the AWGN vector $\|\mathbf{n}\|^{2}$, which is chi-square distributed with mean $N_{r} N_{0}$, variance $N_{r} N_{0}^{2}$ and $2 N_{r}$ degrees of freedom. Now in presence of phase noise,

$$
\|\mathbf{y}-\mathbf{H} \mathbf{x}\|^{2}=\left\|\mathbf{H}_{\theta} \mathbf{x}-\mathbf{H} \mathbf{x}+\mathbf{n}\right\|^{2} .
$$

Now we compute the expected value of the 'impure' ML cost of the correct vector.

$$
\begin{aligned}
& \mathbb{E}\left(\|\mathbf{y}-\mathbf{H} \mathbf{x}\|^{2}\right)=\mathbb{E}\left(\left\|\mathbf{H}_{\theta} \mathbf{x}-\mathbf{H} \mathbf{x}+\mathbf{n}\right\|^{2}\right) \\
= & \mathbb{E}\left(\mathbf{x}^{H}\left(\mathbf{H}_{\theta}-\mathbf{H}\right)^{H}\left(\mathbf{H}_{\theta}-\mathbf{H}\right) \mathbf{x}\right)+\mathbb{E}\left(\|\mathbf{n}\|^{2}\right) \\
= & E_{s} \mathbb{E}\left(\operatorname{tr}\left(\left(\mathbf{H}_{\theta}-\mathbf{H}\right)^{H}\left(\mathbf{H}_{\theta}-\mathbf{H}\right)\right)\right)+N_{r} N_{0} \\
& =2 E_{s} \mathbb{E}(\operatorname{tr}(\mathbf{R}))\left(1-e^{-\sigma_{\theta}^{2}}\right)+N_{r} N_{0} \\
= & 2 E_{s} N_{t} N_{r}\left(1-e^{-\sigma_{\theta}^{2}}\right)+N_{r} N_{0}
\end{aligned}
$$

Equation (6) is obtained from (5) as $\mathbb{E}\left(x_{i} x_{k}\right)=0,1 \leq i \neq$ $k \leq N_{t}$. The proof is given in detail in Appendix A. From (8) it can be observed that due to presence of phase noise the expected value of the 'impure' ML cost of the true vector does not become zero even at infinite SNR. It can also be observed that for a given channel, $\mathbb{E}\left(\|\mathbf{y}-\mathbf{H x}\|^{\mathbf{2}}\right)$ increases with $\sigma_{\theta}^{2}$.

\section{A. ML solution correctness criterion based on norm differ-} ence

Now we investigate whether it is possible to claim that the 'impure' ML solution is the actually transmitted vector or not. In order to do that we study the properties of the norm difference between $\mathbf{x}$ and the best vector among the rest. We define $\mathbf{x}_{M L}^{(2)}$ as the vector having the minimum 'impure' $\mathrm{ML}$ cost among all possible vectors excluding $\mathbf{x}$,

$$
\mathbf{x}_{M L}^{(2)}=\arg \min _{\widetilde{\mathbf{x}} \in \mathcal{C}^{N_{t}}, \widetilde{\mathbf{x}} \neq \mathbf{x}}\|\mathbf{y}-\mathbf{H} \widetilde{\mathbf{x}}\|^{2} .
$$

Note that, when $\mathbf{x}_{M L}^{(1)} \neq \mathbf{x}, \mathbf{x}_{M L}^{(2)}=\mathbf{x}_{M L}^{(1)}$. We are interested in the metric $\kappa$ defined as follows

$$
\kappa \triangleq\left\|\mathbf{y}-\mathbf{H x}_{M L}^{(2)}\right\|^{2}-\|\mathbf{y}-\mathbf{H} \mathbf{x}\|^{2}
$$

Hence, when $\mathbf{x}_{M L}^{(1)}=\mathbf{x}, \kappa>0$, and it is the difference between the two best vectors in terms of 'impure' ML cost. On the other hand, when $\mathbf{x}_{M L}^{(1)} \neq \mathbf{x}, \kappa<0$, and it is the norm difference between actually transmitted vector and the 'impure' ML solution. Now we intend to determine a threshold $\tau$ such that

$$
p\left(|\kappa|<\tau \mid \mathbf{x}_{M L}^{(1)} \neq \mathbf{x}\right) \lesssim 1 .
$$

Hence $\tau$ is the approximate upper bound on the distance between the 'impure' ML vector and the actual vector when they are different. Using this threshold, we generate a candidate set $\mathcal{S}$ whose elements are the data vectors whose 'impure' ML costs are less than $\left\|\mathbf{y}-\mathbf{H} \mathbf{x}_{M L}^{(1)}\right\|^{2}+\tau$.

$$
\mathcal{S} \triangleq\left\{\widetilde{\mathbf{x}}:\|\mathbf{y}-\mathbf{H} \widetilde{\mathbf{x}}\|^{2}<\left\|\mathbf{y}-\mathbf{H} \mathbf{x}_{M L}^{(1)}\right\|^{2}+\tau\right\} .
$$

So the elements of $\mathcal{S}$ are the vectors which when left-multiplied by $\mathbf{H}$ lie inside the $N_{r}$ dimensional hypersphere centred around $\mathbf{y}$ with a radius of $\rho_{\tau} \triangleq \sqrt{\left\|\mathbf{y}-\mathbf{H x}_{M L}^{(1)}\right\|^{2}+\tau}$. The different possible scenarios of 'impure' ML costs of vectors and cardinality of $\mathcal{S}$ are shown in Fig. 1. Let us denote $\mathbf{x}^{*}$ as any point other than $\mathbf{x}$ and $\mathbf{x}_{M L}^{(2)}$. Fig. 1 (a) shows the scenario when there is only point $\mathbf{H} \mathbf{x}_{M L}^{(1)}$ inside the red circle with radius $\rho_{\tau}$. Hence it can be declared to be the correct vector $\mathbf{x}$. The case when $\mathbf{H} \mathbf{x}_{M L}^{(1)}=\mathbf{x}$, but there are other points inside the circle is illustrated in Fig. 1 (b). This is the typical false alarm scenario, when $\mathbf{H x}_{M L}^{(1)}=\mathbf{x}$, but can not be declared to be so. In this case, $|\mathcal{S}| \geq 2$. Fig. 1 (c) shows the scenario when $\mathbf{H x}_{M L}^{(1)} \neq \mathbf{x}$. Also in this case, $|\mathcal{S}| \geq 2$.

Equations (11) and (12) ensures that the candidate set mostly includes the actually transmitted vector, i.e., $p(\mathbf{x} \in \mathcal{S}) \lesssim 1$. Note that, $\mathcal{S}$ is non-empty and contains atleast $\mathbf{x}_{M L}^{(1)}$.

Criterion for declaring $M L$ solution correct: If $\mathcal{S}$ contains only one element, i.e., $\mathbf{x}_{M L}^{(1)}$, then from (11), $p\left(\mathbf{x}_{M L}^{(1)}=\mathbf{x}\right) \lesssim$ 1 , the ML solution obtained ignoring phase noise is mostly the actually transmitted vector.

\section{B. Properties of the norm difference $\kappa$}

To study the properties of $\kappa$, we investigate the ML cost difference between $\mathbf{x}$ and its closest 1-symbol neighbours. We 


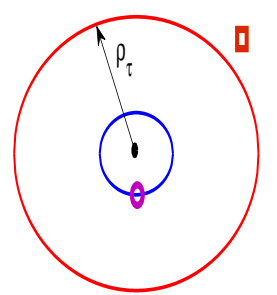

(a)

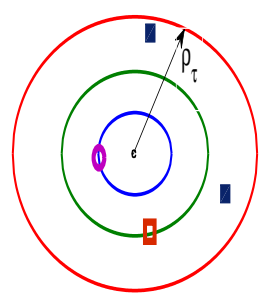

(b)

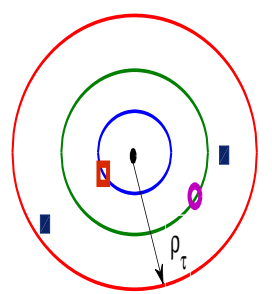

(c)
Fig. 1. Diagram representing different scenarios of ML cost distribution: (a) $\mathbf{x}_{M L}^{(1)}=\mathbf{x},|\mathcal{S}|=1$, (b) $\mathbf{x}_{M L}^{(1)}=\mathbf{x},|\mathcal{S}|>1$, and (c) $\mathbf{x}_{M L}^{(1)} \neq \mathbf{x},|\mathcal{S}|>1$.

compute the term $\delta_{i} \triangleq\left\|\mathbf{y}-\mathbf{H}\left(\mathbf{x}+\lambda_{\mathbf{i}} \mathbf{e}_{\mathbf{i}}\right)\right\|^{2}-\|\mathbf{y}-\mathbf{H} \mathbf{x}\|^{2}$, where $\mathbf{e}_{i}$ is the $i$ th column of $N_{t} \times N_{t}$ identity matrix. Note that, for closest 1-symbol neighbours $\lambda_{i}= \pm 2$, or $\pm 2 j$. Now in high SNR region, ignoring AWGN we obtain

$$
\begin{aligned}
\delta_{i} & =\left\|\left(\mathbf{H}_{\theta}-\mathbf{H}\right) \mathbf{x}-\lambda_{i} \mathbf{h}_{i}\right\|^{2}-\left\|\left(\mathbf{H}_{\theta}-\mathbf{H}\right) \mathbf{x}\right\|^{2}, \\
& =\left|\lambda_{i}\right|^{2} r_{i, i}-2 \Re\left\{\lambda_{i} \mathbf{x}^{H}\left(\mathbf{H}_{\theta}-\mathbf{H}\right)^{H} \mathbf{h}_{i}\right\}, \\
& =\left|\lambda_{i}\right|^{2} r_{i, i}-2 \Re\left\{\lambda_{i} \mathbf{x}^{H} \mathbf{H}_{\theta}^{H} \mathbf{h}_{i}\right\}+2 \Re\left\{\lambda_{i} \mathbf{x}^{H} \mathbf{r}_{i}\right\}(13)
\end{aligned}
$$

where $r_{i, i}$ is the $(i, i)$ th element of $\mathbf{R}$, and $\mathbf{r}_{i}$ is the $i$ th column vector of $\mathbf{R}$.

Now we compute the expected value of $\delta_{i}$ for a given channel matrix.

$$
\begin{aligned}
\mathbb{E}\left(\delta_{i} \mid \mathbf{H}\right)= & 2 \mathbb{E}\left(\Re\left\{\lambda_{i} x_{i}^{H}\right\}\right) r_{i, i}+\left|\lambda_{i}\right|^{2} r_{i, i} \\
& -2 \mathbb{E}\left(\Re\left\{\lambda_{i} \mathbf{x}^{H} \mathbf{H}_{\theta}^{H} \mathbf{h}_{i}\right\}\right)
\end{aligned}
$$

We observe that, for general square $M$-QAM alphabet with uniform probability distributions, $\mathbb{E}\left(\Re\left\{\lambda_{i} x_{i}\right\}\right)=-4+\frac{4}{\sqrt{M}}$. Using this, we can simplify (15) as follows

$$
\begin{gathered}
\mathbb{E}\left(\delta_{i} \mid \mathbf{H}\right)=4 r_{i, i}+2 r_{i, i}\left(-4+\frac{4}{\sqrt{M}}\right)\left(1-e^{-\sigma_{\theta}^{2}}\right) \\
\quad=4 r_{i, i}\left(\left(\frac{2}{\sqrt{M}}-1\right)+\left(2-\frac{2}{\sqrt{M}}\right) e^{-\sigma_{\theta}^{2}}\right) .
\end{gathered}
$$

The details of the derivation is given in Appendix B. We observe that, $\mathbb{E}\left(\delta_{i}\right)$ is positive and decreases with $\sigma_{\theta}^{2}$. Also, it decreases with increasing the constellation size $M$. It is to be noted that, $\kappa \leq \min _{1 \leq i \leq N t} \delta_{i}$. Hence

$$
\mathbb{E}(\kappa) \leq \min _{1 \leq i \leq N t} \mathbb{E}\left(\delta_{i}\right) .
$$

In Fig. 2, we plot the simulated probability distribution functions (PDF) of $\kappa,\|\mathbf{y}-\mathbf{H x}\|^{2}$, and $\delta_{4}$ for a fixed $4 \times 4 \mathrm{MIMO}$ channel using 4-QAM modulation alphabet and operating at $30 \mathrm{~dB}$ average received SNR and $\sigma_{\theta}=6^{\circ}$. The channel matrix used is given in Appendix C. It can be observed that if we set $\tau=2.5, p(\mathbf{x} \in \mathcal{S}) \lesssim 1$. Also, if $\mathcal{S}$ has only one element, i.e., $\mathbf{x}_{M L}^{(1)}, p\left(\mathbf{x}_{M L}^{(1)}=\mathbf{x}\right) \lesssim 1$. If we increase the thresh-

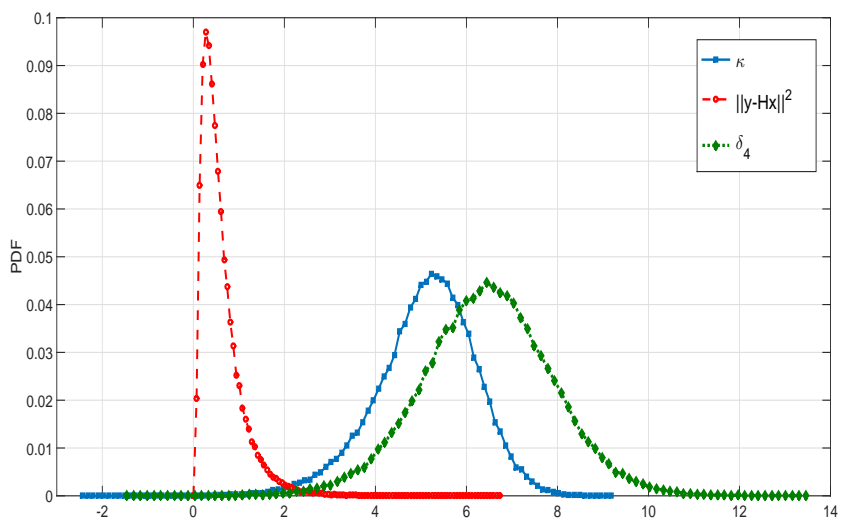

Fig. 2. Probability distribution function of $\kappa,\|\mathbf{y}-\mathbf{H x}\|^{2}$, and $\delta_{4}$ for $4 \times 4$ MIMO, 4-QAM, $30 \mathrm{~dB}$ SNR

old $\tau, p(\mathbf{x} \in \mathcal{S})$ increases. This is beneficial when detected $\mathbf{x}_{M L}^{(1)} \neq \mathbf{x}$, but incurs extra complexity of further processing when $\mathbf{x}_{M L}^{(1)}=\mathbf{x}$ by adding more vectors in $\mathcal{S}$. Hence there is a performance complexity tradeoff in selection of $\tau$.

In Fig. 3, we plot the variation in error in set generation probability i.e., $p\left(\mathbf{x} \notin \mathcal{S} \mid \mathbf{x}_{M L}^{(1)} \neq \mathbf{x}\right)=p\left(|\kappa|>\tau \mid \mathbf{x}_{M L}^{(1)} \neq \mathbf{x}\right)$ and the extra complexity incurred in proportion of cases as $p\left(\kappa \leq \tau \mid \mathbf{x}_{M L}^{(1)}=\mathbf{x}\right)$ with respect to $\tau$. For example, a choice of $\tau=2.5$, results in missing out nearly $5 \%$ error events but at the cost of incurring extra complexity only in close to $7 \%$ cases where ML detection ignoring phase noise itself gives correct solution.

The value of $\tau$ for the purpose of simulation is chosen by observing the histogram of $\kappa$ over different channel realizations beforehand. Hence, it does not need to change for each channel. However, in case of MIMO channels when channel remains constant for a long time, for example LOS MIMO channels, a better value of $\tau$ can be predicted only for that channel.

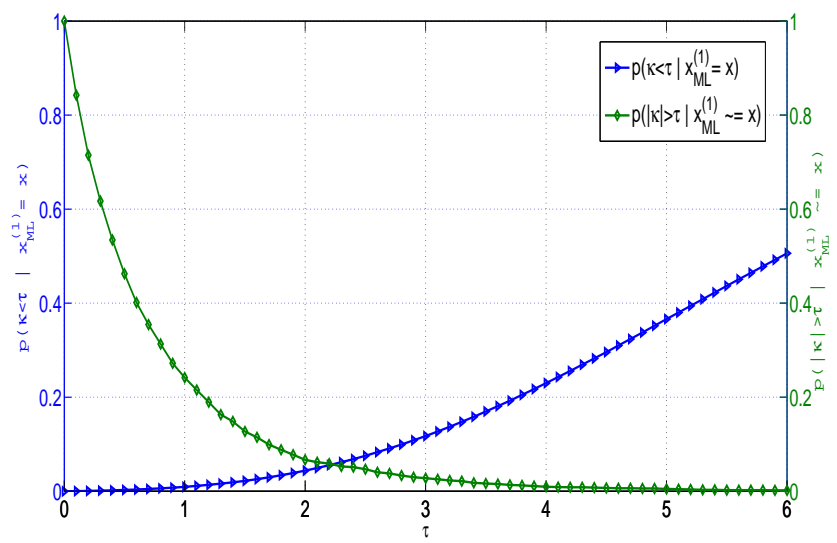

Fig. 3. Error (green line) and complexity (blue line) variation with $\tau$. 


\section{Proposed DATA DETECTION ALGORITHM USING PHASE NOISE ESTIMATION}

The 'impure' $M L$ detection process of evaluating $\mathbf{x}_{M L}^{(1)}$ is suboptimum and suffers from severe performance loss in higher SNR regime as its symbol error rate exhibits error floor. In this section we propose a detection algorithm using the candidate set $\mathcal{S}$ which improves the SER performance.

The true ML criteria for data detection in presence of phase noise can be rewritten as

$$
\begin{aligned}
\mathbf{x}_{M L} & =\arg \max _{\widetilde{\mathbf{x}} \in \mathcal{C}^{N_{t}}} \int_{\widetilde{\theta}} p(\mathbf{y} \mid \widetilde{\mathbf{x}}, \widetilde{\theta}) p(\widetilde{\theta}) d \widetilde{\theta} \\
& \approx \arg \max _{\widetilde{\mathbf{x}} \in \mathcal{C}^{N_{t}}} \max _{\widetilde{\theta}} p(\mathbf{y} \mid \widetilde{\mathbf{x}}, \widetilde{\theta}) p(\widetilde{\theta}) \\
& =\arg \max _{\widetilde{\mathbf{x}} \in \mathcal{C}^{N_{t}}} \max _{\widetilde{\theta}} e^{-\frac{\left\|\mathbf{y}-\widetilde{\mathbf{H}_{\theta}} \tilde{\mathbf{x}}\right\|^{2}}{N_{0}}} e^{-\frac{\sum_{k=1}^{N_{t}+N_{r}} \widetilde{\theta}_{k}^{2}}{2 \sigma_{\theta}^{2}}} \\
& =\arg \min _{\widetilde{\mathbf{x}} \in \mathcal{C}^{N_{t}}} \min _{\widetilde{\theta}}\left\|\mathbf{y}-\widetilde{\mathbf{H}}_{\theta} \widetilde{\mathbf{x}}\right\|^{2}+r \sum_{k=1}^{N_{t}+N_{r}} \widetilde{\theta}_{k}^{2}(20)
\end{aligned}
$$

where in (19) $\widetilde{\mathbf{H}}_{\theta}=\widetilde{\boldsymbol{\Theta}}_{R} \mathbf{H} \widetilde{\boldsymbol{\Theta}}_{T}$. The diagonal matrices $\widetilde{\boldsymbol{\Theta}}_{R}$ and $\widetilde{\boldsymbol{\Theta}}_{T}$ are formed using the entries of the test phase noise vector $\widetilde{\theta}=\left[\widetilde{\theta}_{t, 1}, \widetilde{\theta}_{t, 2}, \cdots, \widetilde{\theta}_{t, N_{t}}, \widetilde{\theta}_{r, 1}, \widetilde{\theta}_{r, 2}, \cdots, \widetilde{\theta}_{r, N_{r}}\right]^{T}$. The validity of the approximation in (18) is discussed in detail in Appendix D. The parameter $r=\frac{N_{0}}{2 \sigma^{2}}$ goes to zero as SNR increases. We define the term in (20) as modified ML cost of the test vector $\widetilde{\mathbf{x}}$ as

$C_{M L}(\widetilde{\mathbf{x}})=\min _{\widetilde{\theta}}\left(\left\|\mathbf{y}-\widetilde{\mathbf{H}}_{\theta} \widetilde{\mathbf{x}}\right\|^{2}+r \sum_{k=1}^{N_{t}} \widetilde{\theta}_{t, k}^{2}+r \sum_{i=1}^{N_{r}} \widetilde{\theta}_{r, i}^{2}\right)$.

Now we propose a approximate method of finding $C_{M L}(\widetilde{\mathbf{x}})$ by computing the optimum $\widetilde{\boldsymbol{\Theta}}_{R}$ and $\widetilde{\boldsymbol{\Theta}}_{T}$ iteratively, rather than jointly which is computationally infeasible. Let us denote $\mathbf{w}=\widetilde{\mathbf{H}} \widetilde{\Theta}_{T} \widetilde{\mathbf{x}}$. The term $\left\|\mathbf{y}-\widetilde{\mathbf{H}}_{\theta} \widetilde{\mathbf{x}}\right\|^{2}$ can be simplified as follows:

$$
\begin{aligned}
\left\|\mathbf{y}-\widetilde{\mathbf{H}}_{\theta} \widetilde{\mathbf{x}}\right\|^{2} & =\left\|\mathbf{y}-\widetilde{\boldsymbol{\Theta}}_{R} \mathbf{w}\right\|^{2} \\
& =\|\mathbf{y}\|^{2}+\|\mathbf{w}\|^{2}-2 \Re\left(\mathbf{y}^{H} \widetilde{\boldsymbol{\Theta}}_{R} \mathbf{w}\right)
\end{aligned}
$$

From (21) it can be observed that the optimum value of entries of $\widetilde{\boldsymbol{\Theta}}_{R}$ for minimizing $\left\|\mathbf{y}-\widetilde{\mathbf{H}}_{\theta} \widetilde{\mathbf{x}}\right\|^{2}$ is given by

$$
\widetilde{\theta}_{r, i}^{o p t}=\angle y_{i}-\angle w_{i}, \forall 1 \leq i \leq N_{r}
$$

Using the optimum $\widetilde{\boldsymbol{\Theta}}_{R}$ in $\left\|\mathbf{y}-\widetilde{\mathbf{H}}_{\theta} \widetilde{\mathbf{x}}\right\|^{2}$, we get

$$
\left\|\mathbf{y}-\widetilde{\mathbf{H}}_{\theta} \widetilde{\mathbf{x}}\right\|^{2}=\|\mathbf{y}\|^{2}+\|\mathbf{w}\|^{2}-2 \sum_{i=1}^{N_{r}}\left|y_{i}\right|\left|w_{i}\right|,
$$

which only depends upon $\widetilde{\boldsymbol{\Theta}}_{T}$, and not $\widetilde{\boldsymbol{\Theta}}_{R}$.

Now, we minimize modified ML cost only with respect to $\widetilde{\Theta}_{T}$. This is done by optimizing each index at a time by keeping other indices fixed. We begin with $\widetilde{\boldsymbol{\Theta}}_{T}=\mathbf{0}_{N_{t} \times N_{t}}$. At index $k$, we compute the term

$\left(\left\|\widetilde{\mathbf{H}} \widetilde{\boldsymbol{\Theta}}_{T} \widetilde{\mathbf{x}}\right\|^{2}-2 \sum_{i=1}^{N_{r}}\left|y_{i} \|\left(\widetilde{\mathbf{H}} \widetilde{\boldsymbol{\Theta}}_{T} \widetilde{\mathbf{x}}\right)_{i}\right|+r \widetilde{\theta}_{t, k}^{2}\right)$ for a set of values for $\widetilde{\theta}_{t, k}$, ranging from $-3 \sigma_{\theta}$ to $3 \sigma_{\theta}$ in steps of $\Delta_{\theta}$. The optimum value is updated at $k$ th index, and we move on to next index. This procedure is repeated for all indices for a certain number of iterations. The final solution of $\widetilde{\boldsymbol{\Theta}}_{T}$ is then used to compute $\widetilde{\boldsymbol{\Theta}}_{R}$ as described in (21). Now using $\widetilde{\boldsymbol{\Theta}}_{T}$ and $\widetilde{\boldsymbol{\Theta}}_{R}$, we compute the term $C_{M L}^{*}(\widetilde{\mathbf{x}}) \triangleq\left\|\mathbf{y}-\widetilde{\mathbf{H}}_{\theta} \widetilde{\mathbf{x}}\right\|^{2}+$ $r \sum_{k=1}^{N_{t}} \widetilde{\theta}_{t, k}^{2}+r \sum_{i=1}^{N_{r}} \widetilde{\theta}_{r, i}^{2}$, referred to as approximate modified ML cost for vector $\widetilde{\mathbf{x}}$. This process is repeated from all entries of candidate set $\mathbb{S}$ and the final detector output can be written as

$$
\mathbf{x}^{\text {det }}=\arg \min _{\widetilde{\mathbf{x}} \in \mathcal{S}} C_{M L}^{*}(\widetilde{\mathbf{x}})
$$

It can be observed that, this method of computing $C_{M L}^{*}(\widetilde{\mathbf{x}})$ is approximate as we do not perform joint optimization over $\widetilde{\boldsymbol{\Theta}}_{R}$ and $\widetilde{\boldsymbol{\Theta}}_{T}$ and also do not take into account $r \sum_{i=1}^{N_{r}} \widetilde{\theta}_{r, i}^{2}$ while computing $\widetilde{\theta}_{r, i}^{\text {opt }}$ to keep the computations tractable. Neglecting $r \sum_{i=1}^{N_{r}} \widetilde{\theta}_{r, i}^{2}$ is a valid approximation when $r<<1$, i.e. No $<<2 \sigma_{\theta}^{2}$ which occurs at high SNR. Hence this method of finding $\mathbf{x}^{\text {det }}$ is expected to have performance loss in low to medium SNR regime.

Next, we propose another heuristic technique which combines the approximate modified ML cost $C_{M L}^{*}(\widetilde{\mathbf{x}})$ and 'impure' ML cost $f(\widetilde{\mathbf{x}})$ to get a more robust metric which will give improved performance in all SNR regime. Let us define

$$
g(\widetilde{\mathbf{x}})=q * f(\widetilde{\mathbf{x}})+C_{M L}^{*}(\widetilde{\mathbf{x}}),
$$

where $q$ is the relative weight. Intuitively, we would prefer $f(\widetilde{\mathbf{x}})$ in low SNR regime, hence $q>1$, and prefer $C_{M L}^{*}(\widetilde{\mathbf{x}})$ in high SNR, making $q<1$. Using this logic and proper scaling we set

$$
q=\frac{N_{0} \min _{\widetilde{\mathbf{x}} \in \mathcal{S}} C_{M L}^{*}(\widetilde{\mathbf{x}})}{2 \sigma_{\theta}^{2} \min _{\widetilde{\mathbf{x}} \in \mathcal{S}} f(\widetilde{\mathbf{x}})} .
$$

detector output can be written as

$$
\mathbf{x}_{n e w}^{\text {det }}=\arg \min _{\widetilde{\mathbf{x}} \in \mathcal{S}} g(\widetilde{\mathbf{x}}) .
$$

The steps of the proposed detection algorithm are listed below:

1) Perform 'impure' $M L$ detection, generate $\mathbf{x}_{M L}^{(1)}$.

2) Generate set $\mathcal{S}$.

3) If $|\mathcal{S}|=1$, declare $\mathbf{x}_{M L}^{(1)}$ to be the correct solution. End.

4) Else, for each $\widetilde{\mathbf{x}} \in \mathcal{S}$, compute $\widetilde{\boldsymbol{\Theta}}_{T}$ and $\widetilde{\boldsymbol{\Theta}}_{R}$. Hence compute $C_{M L}^{*}(\widetilde{\mathbf{x}})$ and then $g(\widetilde{\mathbf{x}})$.

5) Find $\mathbf{x}_{\text {new }}^{\text {det }}$. End.

\section{Simulation Results AND Discussions}

In this section, we present simulation results for different MIMO systems with varying number of antennas and modulation alphabet. In Fig. 4, we present the SER results for $2 \times 2$ and $4 \times 4$ MIMO systems using 4-QAM modulation alphabet. It can be observed that the proposed algorithm is able to achieve better performance in high SNR regime than ML detection ignoring phase noise. The performance improvement in obtained error floor is about about $70 \%$ of the ML detection performance. 


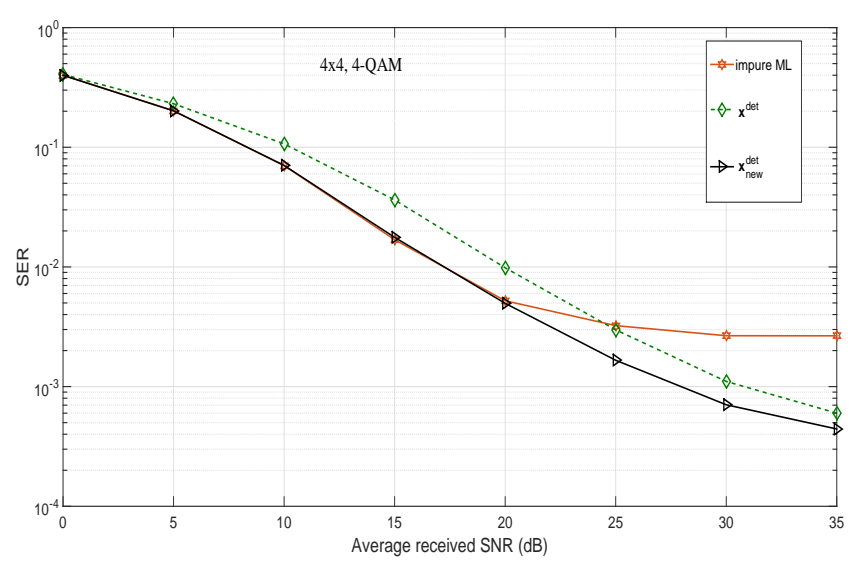

Fig. 4. Symbol error rate for $4 \times 4 \mathrm{MIMO}, 4-\mathrm{QAM}, \sigma_{\theta}=6^{\circ}$.

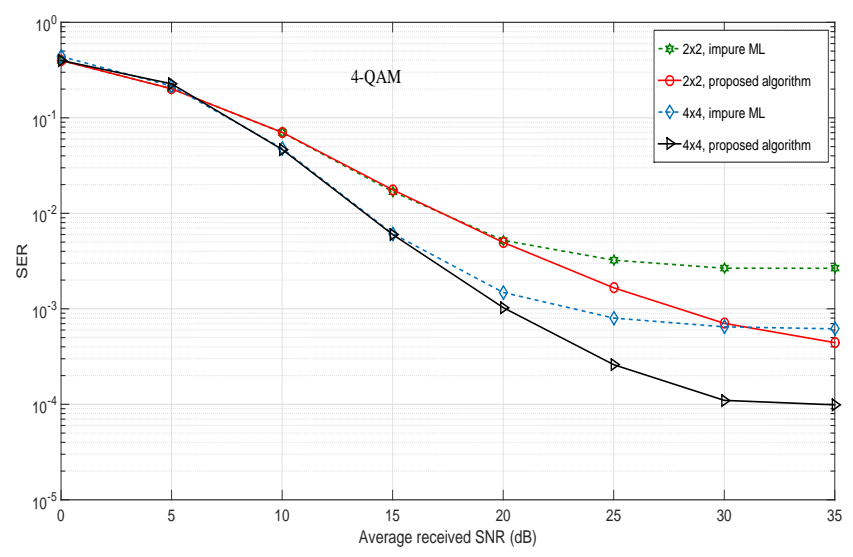

Fig. 5. Symbol error rate for $2 \times 2$ and $4 \times 4$ MIMO, 4-QAM, $\sigma_{\theta}=6^{\circ}$.

\section{Conclusion}

In this paper, we have studied the properties of ML cost function in MIMO systems in presence of phase noise. Our main contribution is to use the traditional ML detection solution which is obtained ignoring phase noise for further processing by estimating phase noise matrices to improve the detection performance. First, we have found a criterion based on norm differences between the best and second best vectors to decide upon the correctness of the ML solution, and also to generate a set of candidate vectors for further processing to improve the solution. Secondly, we have proposed a detection algorithm which performs phase noise estimation for each candidate vector and using the estimated phase noise matrices a modified ML cost is computed. The vector that minimizes the modified ML cost is declared as the final solution. Theoretical arguments have been presented along with SER simulation results for various antenna, modulation alphabet combinations to show the performance improvement obtained by the proposed algorithm.

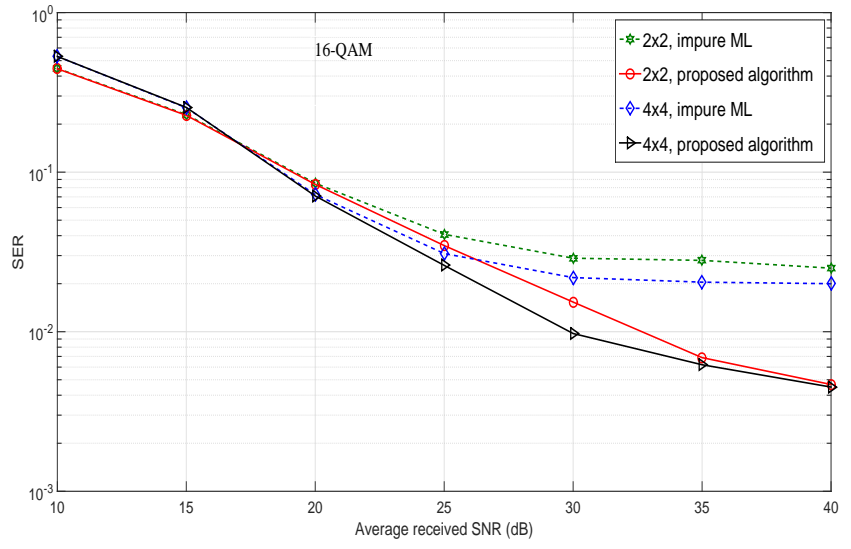

Fig. 6. Symbol error rate for $2 \times 2$ and $4 \times 4$ MIMO, 16-QAM, $\sigma_{\theta}=4^{\circ}$.

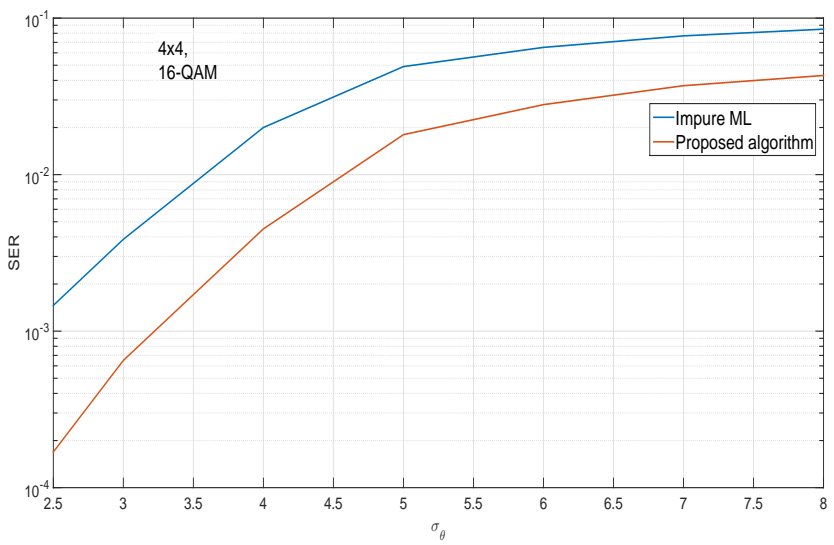

Fig. 7. Symbol error rate for $4 \times 4$ MIMO, 16-QAM, $40 \mathrm{~dB}$ SNR, $\sigma_{\theta}$ variation.

\section{APPENDIX A}

APPENDIX B

APPENDIX C

APPENDIX D

\section{REFERENCES}

